Annales de Parasitologie (Paris), 1975, t. 50, n 1, pp. 105 à 122

\title{
Sur quelques Simulies (Diptera, Simuliidae) du Nord-Ouest de l'Espagne
}

\author{
par F. BEAUCOURNU-SAGUEZ \\ Laboratoire de Parasitologie ( $\mathrm{P}^{\mathrm{r}}$ J.-M. DoBY), Faculté de médecine, F 35000 Rennes
}

\section{Résumé.}

Au cours d'une rapide prospection dans le Nord-Ouest de l'Espagne (Provinces de Santander, Oviedo, León, Lugo, Orense, Zamora et Burgos), nous avons récolté 17 espèces de Simulies dont 8 sont, à notre avis, nouvelles pour ce pays : $S$. (E.) naturale Davies 1966, S. (E.) armoricanum Doby et David 1961, S. (E.) latinum (Rubzov 1962), S. (E.) latizonum (Rubzov 1956), S. (E.) latigonium (Rubzov 1956), S. (O.) spinosum Doby et Deblock 1957, S. (Odagmia) sp. (species propria?), S. (S.) erythrocephalum De Geer 1776.

\section{Summary.}

Studies on some black-flies (Diptera, Simuliidae) of the north-west of Spain.

A collection of Simuliidae was made in the north-west of Spain in the Provinces of Santander, Oviedo, León, Lugo, Orense, Zamora and Burgos. 17 species were found of which 8 were, to our knowledge, new to this country: $S$. (E.) naturale Davies $1966, S$. (E.) armoricanum Doby and David 1961, S. (E.) latinum (Rubzov 1962), S. (E.) latizonum (Rubzov 1956), S. (E.) latigonium (Rubzov 1956), S. (O.) spinosum Doby and Deblock 1957. S. (Odagmia) sp. (species propria?), S. (S.) erythrocephalum De Geer 1776.

\section{Introduction}

Après deux séries de récoltes de simulies dans la Péninsule Ibérique, au Sud du Portugal et dans la partie Sud-Est de l'Espagne (Beaucournu-Saguez, 1972; sous presse), nous avons effectué de nouvelles prospections au Nord-Ouest de ce dernier pays, à l'intérieur d'un quadrilatère limité au Nord et à l'Ouest par l'Atlantique, au Sud par la frontière du Portugal et à l'Est approximativement par la ville de Burgos. 
Nous avons examiné 21 gîtes. Neuf situés dans la région des «Picos de Europa » (Province de Santander, Oviedo et León) et 3 à peu de distance de la mer (Province d'Oviedo) sont dans une région déjà étudiée par Grenier et Bertrand (1954). Les 9 autres gîtes se répartissent dans les provinces de Lugo, Orense, Zamora et Burgos.

Nos observations sont loin d'être complètes. Elles nous ont cependant permis de prospecter des stations de montagne, de plateau et de basse altitude et d'avoir ainsi un échantillonnage d'un certain nombre de biotopes de cette région. Ces nouvelles recherches très limitées dans le temps (du 15 septembre au 4 octobre 1973) ne peuvent être représentatives de toute la faune simulidienne de la Cordilière cantabrique et de son pourtour.

Grenier et Bertrand (op. cit.) qui, à notre connaissance, sont les seuls auteurs à avoir effectué des prélèvements de simulies dans cette région, en citent 8 espèces:

Prosimulium hirtipes (Fries 1824).

Simulium (Eusimulium) aureum Fries 1824.

S. (Tetisimulium) bezzii (Corti 1916). 1920.

S. (Simulium) monticola Friederichs

A l'époque, toutes ces espèces étaient nouvelles pour l'Espagne à l'exception de S. bezzii déjà signalée par Edwards (1933) « en Aragon (à Albarracin)».

Nous avons retrouvé le complexe aureum, $S$. bezzii, $S$. monticola et $S$. variegatum. Nous signalons, en outre, l'existence de :

* S. (E.) naturale Davies 1966.

S. (Obuchovia) auricoma Meigen 1818.

S. (E.) cryophilum (Rubzov 1959).

* $S$. (E.) armoricanum Doby et David 1961.

* S. (E.) latinum (Rubzov 1962).

*S. (E.) latizonum (Rubzov 1956).

* S. (E.) latigonium (Rubzov 1956).

S. (Wilhelmia mediterraneum Puri 1925.

Les 8 espèces précédées d'un astérisque sont nouvelles pour l'Espagne.

\section{Gîtes et matériel étudiés}

1. - Province de Santander, commune de Cervatos. Sur la Nationale 611, $5 \mathrm{~km}$ au sud de Reinosa. Altitude $880 \mathrm{~m}$; grand ruisseau $(5 \mathrm{~m}$ de largeur $\times 0,30 \mathrm{~m}$ Je profondeur); courant $++(1)$; végétation +++ ; fréquence en simulies ++ .

$S$. ornatum: larves et nymphes. 
2. - Palencia, Puerto de Piedrasluengas. Altitude $1365 \mathrm{~m}$; petit torrent coulant en alpage $(2 \times 0,20)$; courant +++ ; végétation + ; fréquence + .

S. cryophilum: 1 larve.

$S$. monticola: quelques larves et nymphes.

3. - Santander, Espinama. Lieu-dit Fuente Dé, $25 \mathrm{~km}$ à l'Ouest de Potes. Altitude $1200 \mathrm{~m}$; rigole-déversoir d'une source captée, à fond de terre et de pierres $(0,20 \times 0,05)$; courant + ; sans végétation (rares feuilles mortes).

Groupe latipes: 5 larves.

4. - Santander, Turieno. Rio Deva, $3 \mathrm{~km}$ à l'Ouest de Potes. Altitude $300 \mathrm{~m}$; rivière torrentueuse très ombragée à fond de pierres $(5 \times 0,40)$; courant ++ ; simulies sur des branchages traînant dans le courant; fréquence +++ .

S. monticola: larves et nymphes.

S. variegatum: larves et nymphes.

5. - Santander, La Hermida, Rio Deva dans le défilé de la Hermida. Altitude $250 \mathrm{~m}$; grande rivière torrentueuse à fond de cailloux $(12 \times 1)$; courant +++ à ++++ ; sans végétation; fréquence +++ .

$S$. auricoma: 1 nymphe.

$S$. monticola: larves.

$S$. variegatum: larves et nymphes.

6. - León, Llanares de la Reina. 2 km au Sud-Ouest du Puerto de San Glorio (Nationale 621). Altitude $1500 \mathrm{~m}$; torrent de montagne coulant à travers des alpages $(3 \times 0,20)$; fond de pierres ; courant +++ ; végétation + ; fréquence ++ .

$S$. nitidifrons: quelques larves et nymphes.

S. monticola: larves, une nymphe.

S. variegatum : larves.

7. - León, Riaño. Au croisement des routes du Puerto del Ponton et du Puerto de Panderruedas. Altitude $1200 \mathrm{~m}$ environ; torrent à fond de pierres $(3 \times$ 0,30 ) coulant dans une prairie au fond d'un vallon; courant +++ (prélèvement après violente pluie d'orage); fréquence ++ .

S. cryophilum: 1 larve, 1 nymphe.

$S$. latinum: quelques nymphes.

$S$. latizonum: quelques nymphes.

$S$. nitidifrons: larves et nymphes.

$S$. bezzii : larves et nymphes.

$S$. variegatum: 1 nymphe.

(1) La cotation en croix est celle que nous avons utilisée dans notre travail sur le Portugal en particulier (Beaucournu-Saguez, 1972). 
8. - Oviedo, Covadonga. Altitude $200 \mathrm{~m}$; Rio Covadonga $(8 \times 1)$ à eaux polluées; fond de pierres ; courant ++++ ; sans végétation.

S. ornatum s. 1. : 3 larves.

9. - Oviedo, Covadonga. Déversoir du Lac de la Ercina. Altitude $1200 \mathrm{~m}$; petit ruisseau $(1 \times 0,10)$ à fond de terre et pierres coulant en alpage ; courant ++ ; végétation +++ .

S. aureum s. 1.: 2 larves.

10. - Oviedo, Riberas. Près de Pravia, sur la C 632. Altitude $150 \mathrm{~m}$; ruisseau à fond de terre et de pierres; courant + ; fréquence ++ .

$S$. nitidifrons: larves et nymphes.

11. - Oviedo, Riberas. A $2 \mathrm{~km}$ à l'Est de Pravia sur la route de Valdemora. Altitude $150 \mathrm{~m}$. Ruisseau à fond de pierres, ombragé, en bordure de forêt ; courant ++ ; végétation +++ ; fréquence ++ .

$S$. spinosum: larves et nymphes.

12. - Oviedo, Riberas. Même endroit : prélèvement d'adultes.

$S$. nitidifrons : 5 femelles.

13. - Lugo, Villalba. $3 \mathrm{~km}$ au Nord-Est de Villalba sur la $\mathrm{N} 634$. Altitude $520 \mathrm{~m}$; ruisseau de prairie à fond de terre $(0,80 \times 0,10)$; courant + ; végétation ++++ ; fréquence ++ .

$S$. naturale: larves, 1 nymphe.

S. latigonium : 3 larves.

$S$. spinosum : larves, quelques nymphes.

14. - Orense, Cea. Altitude $600 \mathrm{~m}$; ruisseau ombragé alimentant le lavoir municipal $(0,30 \times 0,10)$; fond de terre ; courant + ; végétation + ; fréquence + .

$S$. naturale: larves, 1 nymphe.

$S$. aureum s. 1.: 1 larve.

15. - Orense, Cea. Lieu-dit «Fondo de Cea». Altitude $600 \mathrm{~m}$; ruisseau à fond de terre $(1,5 \times 0,20)$; courant ++ ; végétation ++++ ; fréquence + .

$S$. naturale: quelques larves.

$S$. latigonium: larves, 1 nymphe.

16. - Orense, Cea. Monastère de Osera. Altitude $650 \mathrm{~m}$; petite rivière à fond de terre et de pierres $(3 \times 0,30)$; très ombragé ; courant +++ ; végétation ++ ; fréquence ++ .

$S$. armoricanum: larves et nymphes.

$S$. aureum s. 1.: 1 nymphe.

$S$. dorieri : larves et nymphes. 
17. - Zamora, Ribadelago de Franco. Rio Tera, $6 \mathrm{~km}$ au Sud de Ribadelago en aval du Lac de Sanabria. Altitude $1000 \mathrm{~m}$; torrent à fond pierreux $(4 \times 0,50)$; courant +++ ; végétation ++ ; fréquence +++ .

$S$. nitidifrons: nombreuses larves et nymphes.

$S$. (Odagmia) sp. : larves et nymphes.

18. - Zamora, Ribadelago de Franco. $3 \mathrm{~km} \mathrm{au} \mathrm{Nord,} \mathrm{en} \mathrm{amont} \mathrm{du} \mathrm{Lac} \mathrm{de}$ Sanabria. Altitude $1000 \mathrm{~m}$; très petit torrent à fond pierreux $(0,50 \times 0,10)$; courant ++ ; sans végétation; débris végétaux ++ ; fréquence + .

$S$. nitidifrons: larves et nymphes.

19. - Zamora, Ribadelago de Franco. Même endroit: Rio Tera. Torrent à fond pierreux $(3 \times 0,50)$; courant +++ ; débris végétaux ++ ; fréquence +++ .

$S$. nitidifrons : larves et nymphes.

$S$. (Odagmia) sp. : larves et nymphes.

20. - Zamora, Camarzana de Tera. $21 \mathrm{~km}$ à l'Ouest de Benavente; ruisseau herbeux à fond de terre $(1,20 \times 0,40)$; courant ++ ; végétation ++++ ; fréquence +++ .

S. latinum: larves et nymphes.

$S$. mediterraneum: larves et nymphes.

$S$. nitidifrons: larves et nymphes.

$S$. erythrocephalum: larves et nymphes.

21. - Province de Burgos. 29,600 km au Sud de Burgos sur la Nationale 1 ; alt. $850 \mathrm{~m}$; petit ruisselet en contrebas de la route, envahi par la végétation $(0,20 \times 0,05)$; fond de graviers et de terre ; courant ++ ; végétation +++++ ; fréquence ++ .

$S$. latigonium: larves et nymphes.

\section{Étude des espèces}

Plusieurs zoologistes (Beirne 1952, Moreau 1954) ont décrit sous le nom de « répartition lusitanienne» des similitudes faunistiques entre la région qui nous occupe et les Iles Britanniques. Sans entrer dans la discussion de ce problème, nous devons souligner les convergences géologiques et climatiques entre ces deux régions entraînant un parallélisme écologique et par conséquent des espèces identiques ou apparentées entre les Iles Britanniques et la région cantabrique. Un certain nombre d'espèces confirment ce fait. Néanmoins, des influences méditerranéennes ou altitudinales vont donner un caractère particulier à la faune que nous avons observée.

Par ailleurs, dans la majorité des gîtes prospectés, l'influence atlantique, dominante sur presque toute cette région, empêche certainement la remontée des espèces éthiopienne et nord-africaine, que nous avons, dans des travaux antérieurs, signalées de la partie sud de la Péninsule ibérique. 
S. (E.) naturale Davies 1966.

Cette espèce isolée du complexe vernum (2), en Angleterre, est très voisine dans l'ensemble de $S$. (E.) vernum Macquart 1826 (3). Selon Davies, les différences essentielles, à part la petite taille, se situent au niveau des filaments respiratoires de la nymphe qui sont minces et dont les deux troncs basaux sont longs, l'inférieur étant deux à trois fois aussi long que le supérieur.

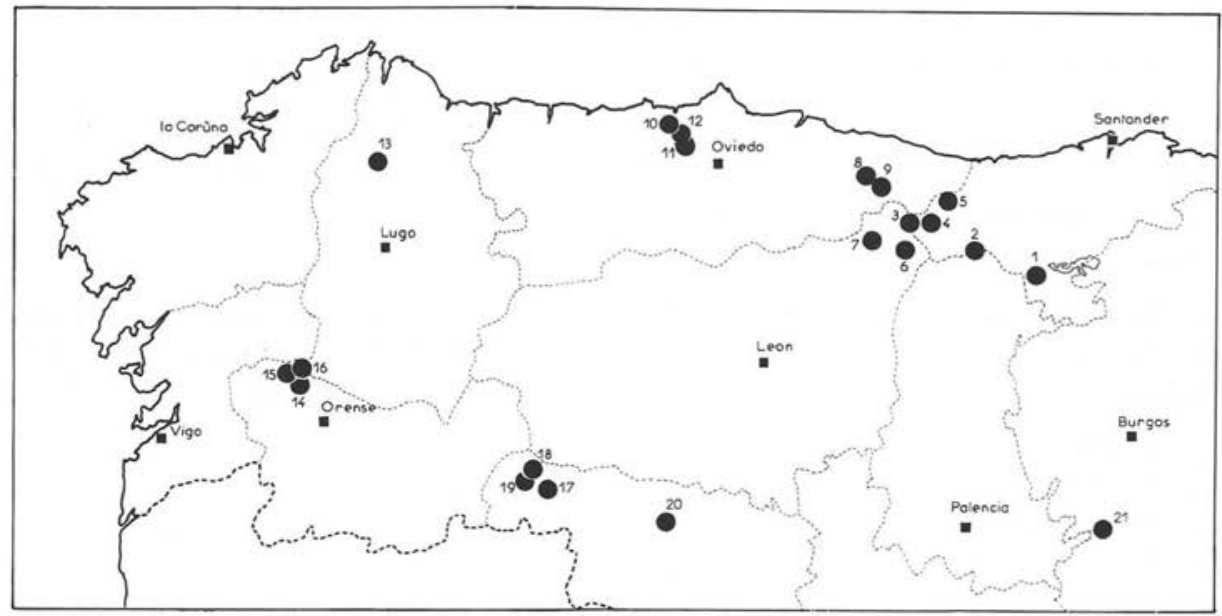

Carte: Emplacement des gîtes prospectés dans le Nord-Ouest de l'Espagne.

Nous avons pu vérifier ce caractère chez nos exemplaires nymphaux qui nous ont frappé par l'aspect grêle et élancé de leurs filaments (fig. 1). Nos prénymphes présentent également les détails morphologiques caractéristiques de l'espèce.

Les trois gîtes dans lesquels nous avons récolté $S$. naturale sont les plus occidentaux de nos prélèvements: Villalba, au Nord de Lugo (gîte 13), et Cea, au NordOuest d'Orense (14 et 15 ). Il s'agit de très petits ruisseaux, à faible altitude (entre 5 et $600 \mathrm{~m}$ ), riches en végétation, où l'eau s'écoule lentement.

$S$. naturale y est associée à $S$. latigonium (3 fois) et $S$. spinosum (1 fois).

L'écologie, la biologie et la distribution géographique de cette espèce demeurent très mal connues. Elle n'avait jusqu'à présent été rencontrée qu'au Nord de l'Angleterre et en Ecosse dans des cours d'eau de terrains marécageux.

\section{S. (E.) cryophilum (Rubzov 1959).}

Cette simulie, commune et à vaste répartition paléarctique y compris la sous-région méditerranéenne, se révèle rare dans nos prélèvements. Nous l'avons récoltée dans

(2) Anciennement complexe latipes (Crooskey et Davies, 1972).

(3) Syn.: S. (E.) latipes sensu auct., non Meigen 1804, Crosskey et Davies 1972. 
deux gîtes (2 et 7) de la chaîne cantabrique, entre 1200 à $1400 \mathrm{~m}$ d'altitude, gîtes qui s'ajoutent à la seule autre station connue de ce pays: Sierra Alta dans la Province de Teruel (Beaucournu-Saguez, sous presse). Il est vraisemblable que des prélèvements effectués au printemps ou au début de l'été permettraient d'élargir la carte de S. cryophilum, espèce apparemment orophile.
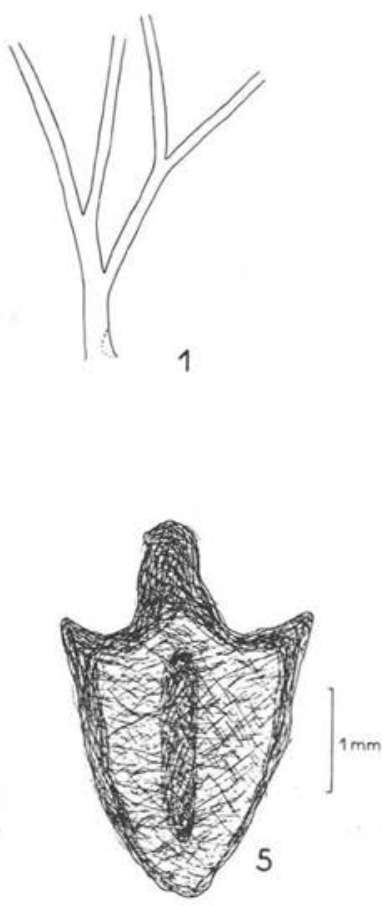

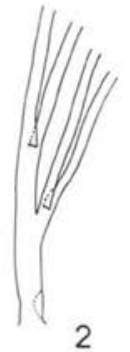

2
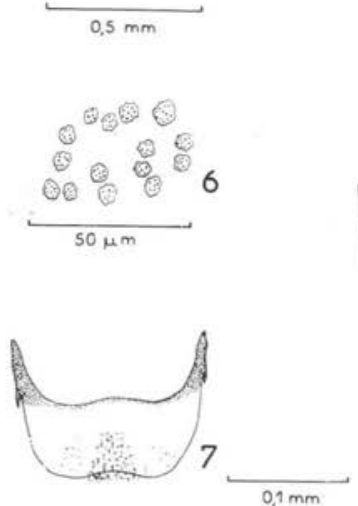
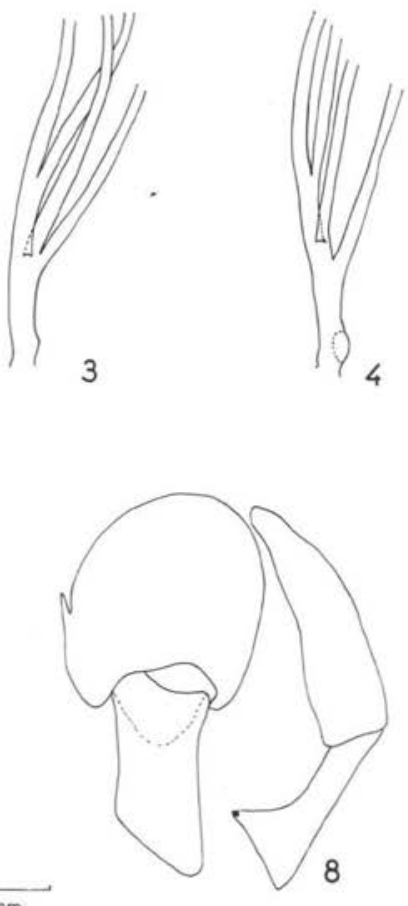

Fig. 1: $S$. naturale, filaments respiratoires de la nymphe (gîte 14). Fig. 2 à $8: S$. armoricanum (gîte 16), 2,3 et 4 : filaments respiratoires de nymphes; 5 : cocon ; 6 : tubercules thoraciques de la nymphe; 7 et 8 : genitalia mâles; $7:$ plaque ventrale; 8 : coxites et styles en vue ventrale et ventro-latérale.

S. (E.) armoricanum Doby et David 1961.

Décrite de l'Ouest de la France (départements du Morbihan et de l'Orne), cette espèce n'a, par la suite, été signalée que d'Angleterre (Doby et Saguez, 1963 ; Davies, 1966). Davies l'a récoltée dans de petits ruisseaux à fond de pierres dans les zones élevées s'étendant de la Cornouaille au Nord-Ouest de l'Ecosse. En France, les quelques stations bretonnes connues sont à $200 \mathrm{~m}$ d'altitude environ; le biotope classique est un petit cours d'eau torrentueux. 
Nous retrouvons de tels caractères dans le gîte 16 (Nord-Ouest d'Orense) situé à $650 \mathrm{~m}$ d'altitude. $S$. armoricanum y est peu abondante, associée à $S$. dorieri, espèce à exigences écologiques voisines, et à une espèce du groupe aureum.

Les stades nymphaux de cette espèce sont très caractéristiques. La diagnose peut en particulier être faite sur le cocon pourvu d'un large processus antérieur, les filaments respiratoires et les tubercules thoraciques de la nymphe (fig. 2 à 8).

\section{S. (E.) latinum (Rubzov 1962).}

S. latinum représente l'espèce du groupe aureum la plus commune dans les pays méditerranéens où ce complexe a été étudié: Algérie (Rivosecchi, 1963), Tunisie (Bailly-Choumara et coll., 1970), Yougoslavie (Zivkovic, 1966), Italie (Rivosecchi, 1963 ; 1967), Sud de la France (Bernard, 1970 ; Jarry, 1973 a). Espèce de petits cours d'eau chauds et lents, de basse altitude, elle peut se maintenir dans des eaux relativement riches en matières organiques (Rivosecchi, 1971 ; Jarry, $1973 a$ ). Cependant, Rivosecchi $(1962 ; 1967 ; 1971)$ l'a rencontrée en semi-altitude $(800$ à $1000 \mathrm{~m})$ en Italie centrale et méridionale.

Au cours de nos prospections, nous l'avons récoltée deux fois (gîtes 7 et 20) (fig. 12 à 17). Le gîte 20 (à l'ouest de Benavente) est un ruisseau, sans ombrage, à eaux polluées, où $S$. latinum est associée à $S$. mediterraneum, $S$. nitidifrons et $S$. erythrocephalum; la station 7 située en bordure sud-ouest des «Picos de Europa », à $1200 \mathrm{~m}$ d'altitude, ne correspond pas au biotope classique de cette espèce. Toutefois, ce prélèvement a été fait après une violente pluie d'orage et sous un mauvais temps persistant qui n'a pas permis de noter les caractéristiques écologiques réelles du gîte. On peut seulement noter que le ruisseau coulait en bordure d'une hêtraie.

\section{S. (E.) latizonum (Rubzov 1956).}

Cette espèce de l'Est et du Sud de l'Europe a d'abord été découverte dans la Province de Léningrad et en Ukraine. Elle a été citée ensuite de Roumanie (Serban, 1961), du Sud de l'Italie et de la Sardaigne (Rivosecchi, 1962 ; Rivosecchi et Solinias, 1962), de Yougoslavie (Rivosecchi, 1963). Elle n'est pas connue d'Algérie (Rivosecchi, 1963), ni de Tunisie (Bailly-Choumara et coll., 1970) où ces auteurs n'ont récolté que $S$. latinum. Par contre, Crosskey (1967) la signale du Moyen-Orient où elle est, avec $S$. (E.) rubzovianum (Serban 1961), le seul représentant connu du groupe aureum.

S. latizonum est nouvelle pour l'Espagne. Un seul prélèvement nous l'a livrée: le gîte 7 , à $1200 \mathrm{~m}$ d'altitude où elle est associée à $S$. latinum. La forme des genitalia mâles ex nympha est en accord avec celle des dessins de Serban (1961) et de Rivosecchi (1962) (fig. 9 à 11). Ce caractère nous paraît être le seul, chez les nymphes, permettant la séparation entre ces deux espèces.

S. (E.) latigonium (Rubzov 1956).

Isolée du complexe angustitarse par Rubzov en Europe septentrionale (région de Léningrad), $S$. latigonium demeure une espèce peu fréquente bien qu'elle ait été signa- 
lée de différents pays de la zone paléarctique: Tchécoslovaquie (Knoz, 1965), Angleterre (Davies, 1966) et Italie (Rivosecchi, 1967).

Nous estimons que les larves de cette espèce sont caractéristiques par leur échancrure ventrale quadrangulaire de type «aureum quoique plus petite. Le bord anté-
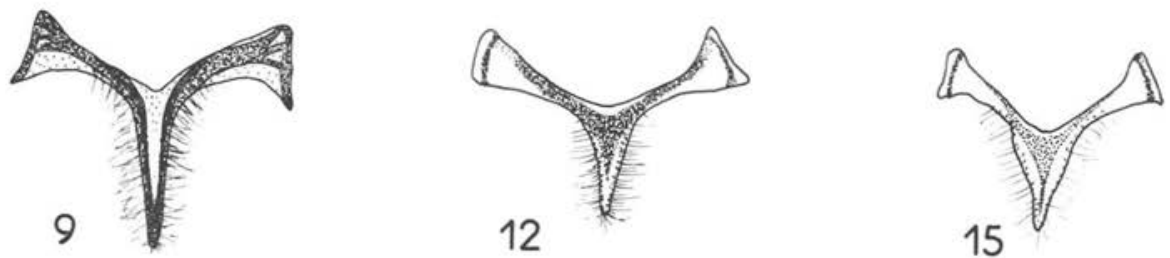
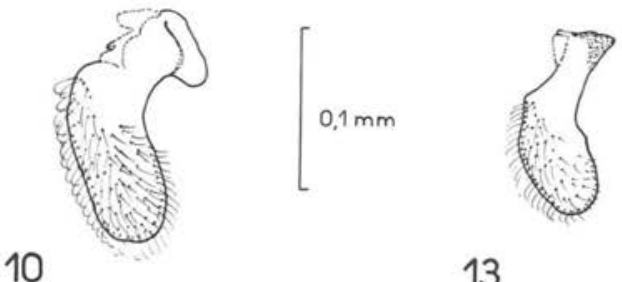

13
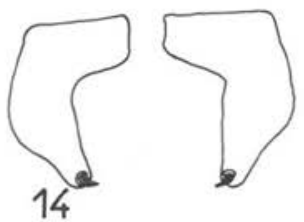

11

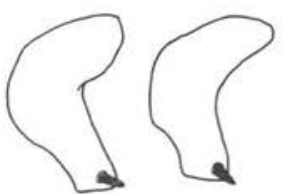

FIG. 9 à 11: Genitalia mâles de S. latizonum (gîte 7). 9: plaque ventrale en vue ventrale; 10 : plaque ventrale en vue latérale; 11 : styles. Fig. 12 à 17 : genitalia mâles de S. latinum; 12 à 14 : gîte $7 ; 15$ à 17 : gîte 20 .

rieur de cette échancrure se contınue par une large tâche pigmentée pouvant s'étendre jusqu'à peu de distance du bord postérieur du submentum. Ce dernier caractère est noté par Knoz et Rivosecchi, mais non par Rubzov (1956) ou Davies. L'ornementation dorsale de la capsule céphalique de nos larves correspond à celle donnée par Rubzov, $\mathrm{Knoz}$ et Rivosecchi.

Les genitalia mâles de nos exemplaires nymphaux ont une morphologie identique à celles figurées par ces auteurs; la plaque ventrale présente une carène fortement proéminente et la plaque dorsale est sensiblement triangulaire (fig. 18 à 24).

Nous avons récolté $S$. latigonium dans les gîtes 13 , 14 et 15 qui sont ceux renfermant $S$. naturale et dans le gîte 21 (Sud de Burgos). Il s'agit de très petits ruisseaux 
riches en végétation et à courant lent, situés à des altitudes inférieures ou égales à $600 \mathrm{~m}$. (1 fois).

Les espèces associées sont $S$. naturale (3 fois), $S$. spinosum et le groupe aureum
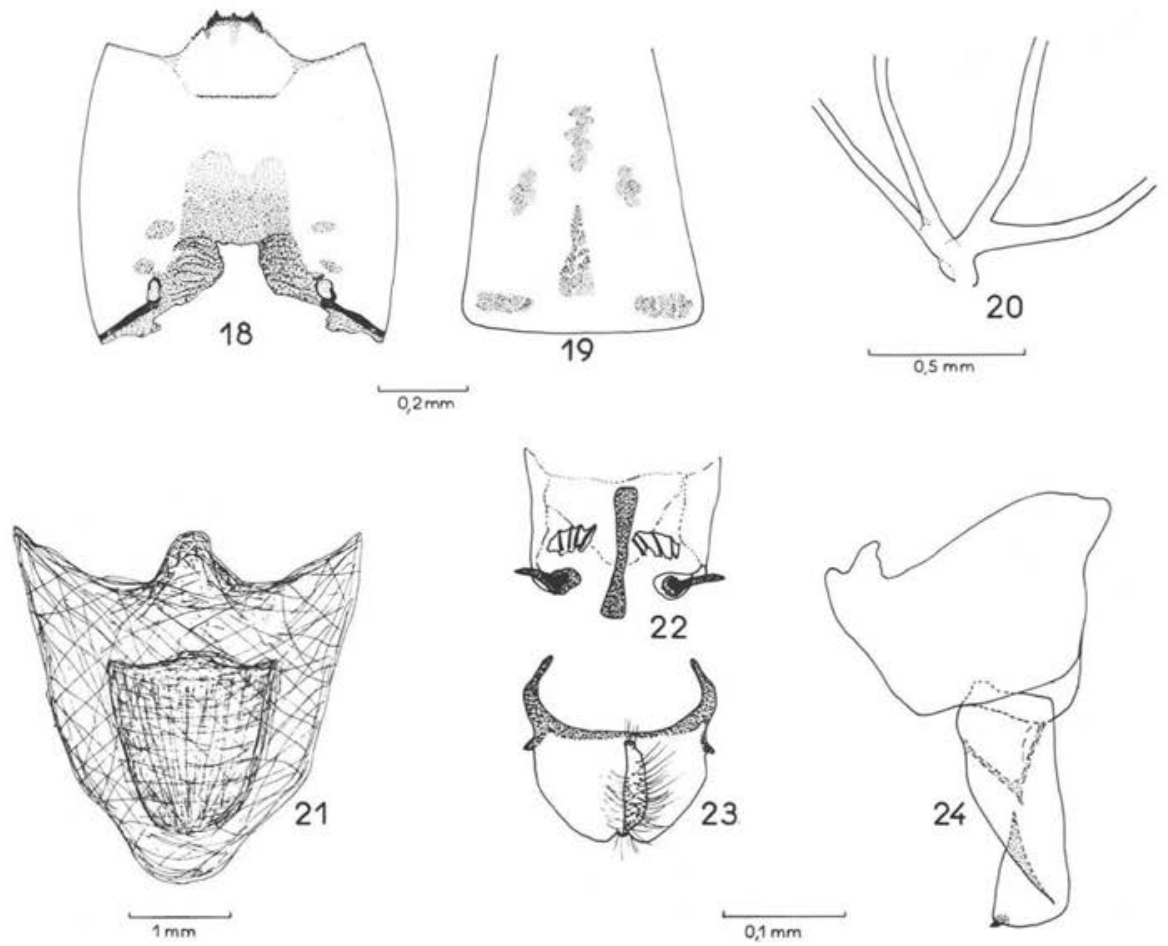

Fig. 18 à $24: S$. latigonium (gîte 21). 18 et 19 : capsule céphalique de la larve en vue ventrale et dorsale ; 20: filaments respiratoires de la nymphe; 21 : cocon; 22 à 24 : genitalia mâles; 22 : plaque dorsale, sclérite médian et paramères; 23 : plaque ventrale ; 24 : coxite et style.

\section{S. (W.) mediterraneum Puri 1925.}

Nous avons déjà souligné que les influences méditerranéennes étaient faibles dans cette partie Nord-Ouest de l'Espagne. Une preuve en est apportée par la rareté de $S$. méditerraneum, très fréquente dans la sous-région méditerranéenne. Elle ne figure que dans un de nos prélèvements (gîte 20), situé au Sud-Est du quadrilatère prospecté. Mais il est possible que la saison, particulièrement froide et pluvieuse, n'ait pas permis, à cette époque de l'année, le développement de cette simulie des cours d'eau chauds et en général peu profonds de plaine ou de basse altitude. 
Dans notre station, cette espèce est associée à $S$. latinum, $S$. nitidifrons et S. erythrocephalum.

\section{S. (Obuchovia) auricoma Meigen 1818.}

Considérée comme une espèce montagnarde de cours d'eau rapides ou même violents (Giudicelli, 1961), elle a cependant été parfois récoltée à basse altitude (dans la région alpine par exemple: Dorier, 1962-1963).

S. auricoma s. sto. a été récoltée et redécrite par Grenier et Bertrand (1949) des Pyrénées-Orientales. Ces mêmes auteurs (1954) l'ont retrouvée dans les massifs pyrénéens espagnols. Notre seule récolte (gîte 5) dans les Picos de Europa $(250 \mathrm{~m}$ d'altitude) permet d'étendre vers l'ouest l'aire de répartition en Espagne de cette simulie.

Nous ne disposons que d'une seule nymphe dont les filaments respiratoires et l'ornementation céphalique et thoracique correspondent à ceux dessinés par Giudicelli (1962).

Espèces associées: $S$. monticola et $S$. variegatum.

\section{Groupe * ornatum *}

Plusieurs espèces de ce complexe très répandu dans toute la région paléarctique sont représentées dans nos prélèvements. En l'absence d'adultes, il est difficile, comme le fait remarquer Crosskey (1967), de faire une identification spécifique. Néanmoins, certaines caractéristiques morphologiques nous sont apparues chez nos exemplaires larvaires et nymphaux qui nous permettent de penser que notre matériel comporte au moins quatre types différents, que nous assimilons à :

S. (Odagmia) ornatum Meigen 1818.

S. (O.) nitidifrons Edwards 1920.

S. (O.) spinosum Doby et Deblock 1957.

S. (Odagmia) sp.

Jusqu'à présent, seules $S$. ornatum et $S$. nitidifrons étaient connues d'Espagne.

1) $\boldsymbol{S}$. (O.) ornatum s. sto. Cette simulie semble rare: nous l'avons trouvée dans un seul gîte (gîte 1) sur les 12 qui contenaient des représentants de ce groupe. Les pièces génitales mâles sont typiques de $S$. ornatum; les exemplaires nymphaux présentent en outre une cuticule céphalique et thoracique pauvre en tubercules (Rivosecchi et Lipparoni, 1964).

2) $S$. (O.) nitidifrons. Beaucoup plus fréquente que la précédente, cette espèce est présente dans 8 de nos prélèvements $(6,7,10,12,17,18,19$ et 20), fréquence qui est en accord avec sa répartition géographique. Elle est en effet toujours largement distribuée dans la sous-région méditerranéenne y compris l'Afrique du Nord. Notre détermination a porté essentiellement sur des stades préimaginaux. Dans le gîte 12 cependant, nous avons capturé cinq femelles à front noir brillant, caractère qui distingue les femelles de $S$. nitidifrons de celles de $S$. ornatum à front gris terne avec quelques soies dorées. 
S. nitidifrons ne montre pas d'affinités écologiques particulières dans les différents gîtes où nous l'avons récoltée. Il s'agit en général de petits ruisseaux ou torrents dont cinq sont situés en altitude (de 1000 à $1500 \mathrm{~m}$ ) et deux à basse altitude dans la zone côtière d'Oviedo.

L'éventail des espèces associées est assez large : S. latinum, S. monticola, S. variegatum, S. (Odagmia) sp. (2 fois), S. latizonum, S. cryophilum, S. bezzii, S. mediterraneum, $S$. erythrocephalum (1 fois).

3) S. (O.) spinosum a d'abord été isolée comme forme de $S$. ornatum par Doby et Deblock (1957), forme basée sur la présence des tubercules épineux qui recouvrent la capsule céphalique et le thorax de la pupe. Knoz (1965) et Davies (1966), s'appuyant sur la structure des genitalia mâles et en particulier de la plaque ventrale, l'ont élevée au rang d'espèce.

Elle a, jusqu'à présent, été signalée de divers pays d'Europe: Autriche, France, Grande-Bretagne, Tchécoslovaquie et Italie. C'est en France (Doby et David, 1960 ; Dorier, 1962-1963 ; Serra-Tosio, 1962-1963 ; Jarry, 1973 b) et en Grande-Bretagne (Doby et Saguez, 1963 ; Davies, 1966 ; Crosskey, 1970) que S. spinosum est la plus fréquente. Cette simulie est certainement plus septentrionale que $S$. nitidifrons.

En Espagne, nous l'avons récoltée dans les gîtes 11 et 13, respectivement à 150 et $520 \mathrm{~m}$ d'altitude. Il s'agit de petits ruisseaux à courant lent et à végétation abondante. Nous n'y avons rencontré que des larves et quelques exuvies nymphales.

$S$. spinosum y est associée à $S$. naturale et à $S$. latigonium (1 fois).

4) S. (Odagmia) sp. (species propria ?). Dans les gîtes 17 et 19, situés sur le Rio Tera, assez voisins géographiquement [l'un (19) en amont du Lac de Sanabria, l'autre (17) en aval] nous avons récolté, associées aux stades immatures de $S$. nitidifrons, des larves et des nymphes présentant des caractères morphologiques particuliers (fig. 25 à 35).

Les larves se distinguent d'emblée par leurs branchies rectales ramifiées: les deux branchies externes présentent près de leur base 3 à 4 petits lobes courts, tandis que la branchie centrale n'en a que deux. On peut cependant observer quelques variations dans le nombre de lobes. L'existence de ces appendices rectaux à digitations secondaires rapproche ces larves de celles de $S$. (O.) frigida (Rubzov, 1956), $S$. (O.) bronchiale (Rubzov, 1962) et $S$. (O.) ornatum (Meigen) subsp. 2 (Rubzov, 1967). On peut exclure les autres espèces ou sous-espèces du groupe «ornatum » de la région paléarctique, qui ont toutes des branchies simples.

Le caractère le plus frappant chez la nymphe est l'absence totale de tubercules sur la cuticule céphalo-thoracique qui, vue à la loupe, a un aspect brillant et lisse (4), cette particularité existant chez $S$. bronchiale; la morphologie cuticulaire n'est pas décrite pour les autres espèces. cules.

(4) A l'inverse, $S$. nitidifrons trouvée dans le même gîte présente une cuticule riche en tuber- 
Les genitalia mâles sont très proches les unes des autres dans ces quatre formes. Il nous semble que c'est de $S$. (O.) ornatum subsp. 2 que nos exemplaires se rapprochent le plus, particulièrement par la forme de la plaque ventrale, le nombre des grandes épines des paramères (cinq) et le profil du style.
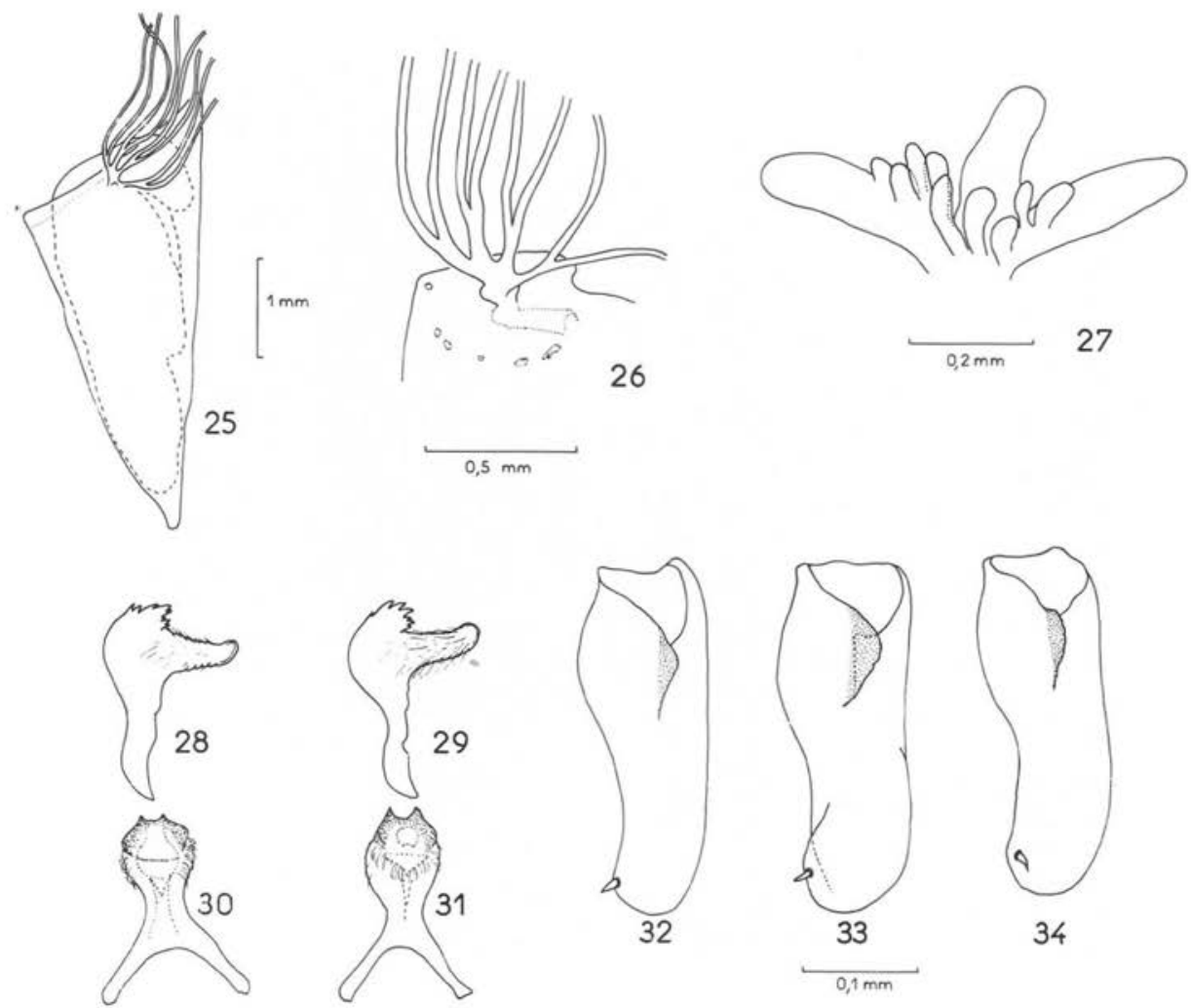

FIG. 25 à $34: S$. (Odagmia) sp. (gîte 17). 25: nymphe ; 26: filaments respiratoires de la nymphe ; 27 : branchies de la larve; 28 à 34 : genitalia mâles; 28 et 29 : plaque ventrale en vue latérale de deux exemplaires; 30 et 31 : id. : en vue ventrale; 32 , 33 et 34 : styles de trois exemplaires.

$S$. (O.) ornatum subsp. 2 a été décrite au mois de mai, dans le Piémont. S. bronchiale n'est connue que de la région de Léningrad et $S$. frigida d'Europe et de Sibérie orientale (teste Rubzov, 1956, 1962).

\section{S. (T.) bezzii Corti 1916.}

$S$. bezzii est répandue dans les pays occidentaux du pourtour méditerranéen: France (Alpes, Pyrénées, Vallée du Rhône, Roussillon et Languedoc), Italie, You- 
goslavie, Algérie et Maroc. Déjà signalée par Edwards (1933) en Espagne, elle y a été retrouvée par Grenier et Bertrand (1954) sur le versant Sud des Pyrénées et dans la Cordillière cantabrique. Nous l'avons nous-mêmes récoltée dans le gîte 7 (1 $350 \mathrm{~m}$ d'altitude) où elle est associée à $S$. cryophilum, $S$. latinum, $S$. latizonum, $S$. nitidifrons et $S$. variegatum.

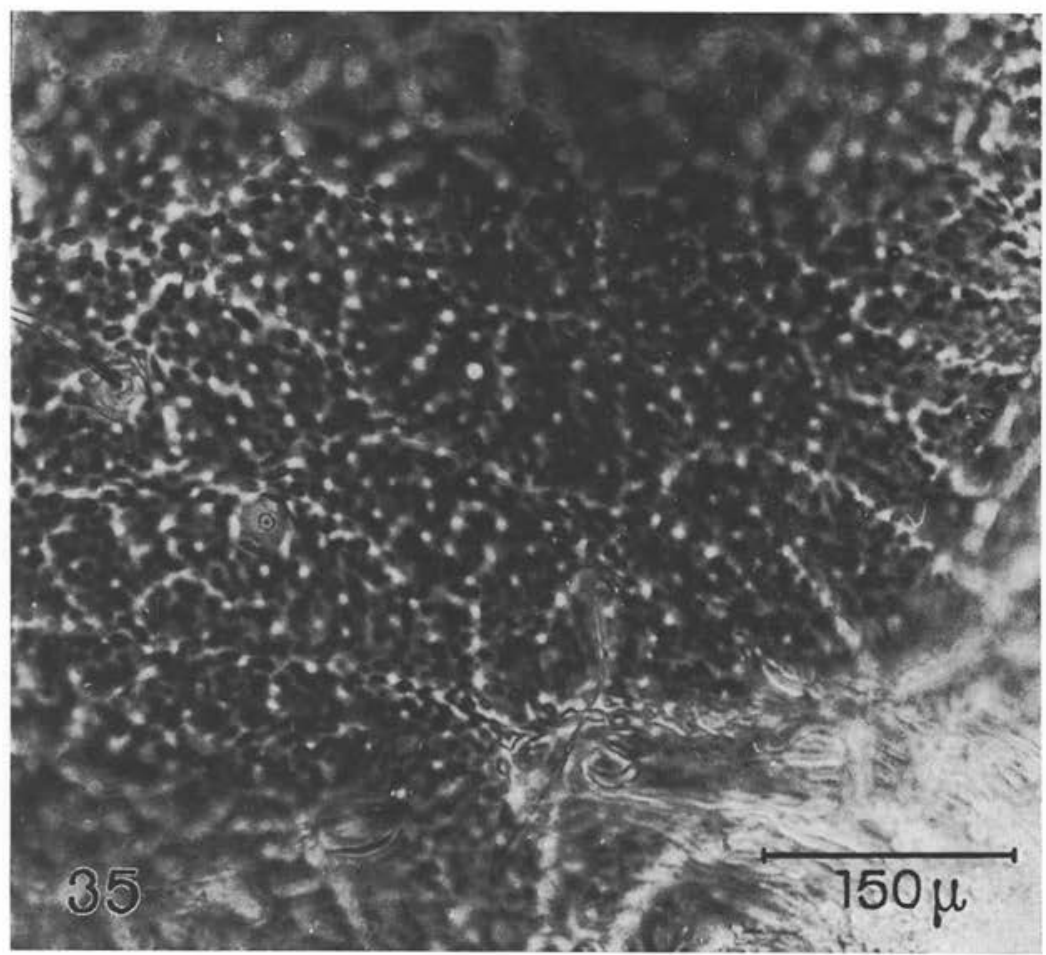

FIG. 35: S. (Odagmia) sp. (gîte 17); aspect cuticulaire thoracique de la nymphe.

Il s'agit d'une espèce eurytherme, fréquente de 0 à $600 \mathrm{~m}$, mais qui peut se rencontrer jusqu'à $2000 \mathrm{~m}$ (Dorier 1962-1963). Dans le Sud de la France, Jarry (1973 a) l'a récoltée souvent en compagnie de $S$. latinum, espèce «la mieux adaptée à l'eutrophie » et capable de se maintenir dans des ruisseaux temporaires en voie d'assèchement.

\section{S. (S.) erythrocephalum De Geer 1776.}

Cette espèce, fréquente dans les cours d'eau de plaine en Europe, semble, cependant, plus rare dans les pays du bassin méditerranéen. Elle a été signalée pour la première fois d'Afrique du Nord en 1970 (Tunisie: Bailly-Choumara et coll.) et 
n'était, jusqu'à présent, pas encore connue de la Péninsule ibérique. En Allemagne, Tchécoslovaquie, Yougoslavie et Italie, c'est une simulie dangereuse par sa piqûre pour l'homme, et surtout les animaux, au moment des sorties massives d'adultes au printemps ou au début de l'été.

Nous ne la signalons que d'un gîte (20), ruisseau herbeux de basse altitude. Les pièces génitales mâles (fig. 36 à 40) correspondent à celles représentées par les
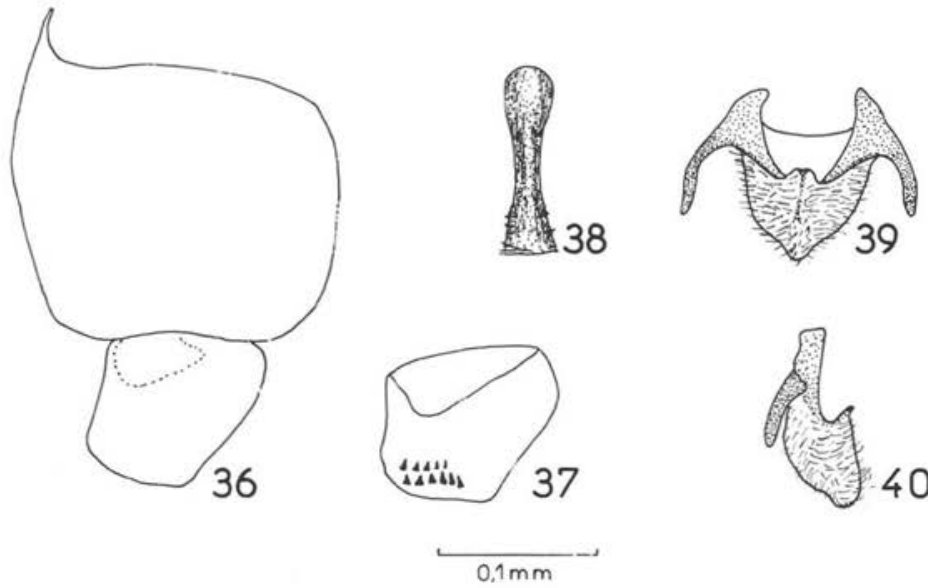

FIg. 36 à 40 : Genitalia mâles de $S$. erythrocephalum; 36 : coxite et style ; 37 : face inférieure du style; 38 : sclérite médian; 39 et 40 : plaque ventrale en vues ventrale et latérale.

divers auteurs (Knoz 1965 ; Davies 1966; Rivosecchi 1967) pour S. erythrocephalum s. sto.

Espèces associées: $S$. latinum, $S$. mediterraneum et $S$. nitidifrons.

\section{Groupe "monticola *}

Simulium monticola a été signalé d'Espagne par Grenier et Bertrand (1954) à une époque où le complexe monticola n'était pas encore scindé. Ces auteurs énumèrent six gîtes de haute altitude dont un dans la Cordillière cantabrique. Personnellement, au cours de nos recherches dans le Sud-Est de ce pays (1975), nous n'avons rencontré que $S$. dorieri Doby et Rault 1960 dans la Sierra Alta à $1600 \mathrm{~m}$ d'altitude. Nos nouvelles récoltes nous ont permis de récolter $S$. monticola Friederichs 1920 et $S$. dorieri, la première étant plus fréquente que la seconde.

i) S. (S.) monticola Friederichs 1920 sensu Doby et Rault, 1960.

Présente dans quatre gîtes $(2,4,5$ et 6$), S$. monticola reste limitée, dans nos récoltes, à la région montagneuse des Picos de Europa (250 à $1500 \mathrm{~m}$ d'altitude). dans des cours d'eau d'aspect torrentueux, à fond de pierres et bien oxygénés. 
Elle suit de très près la distribution de $S$. variegatum à laquelle elle est associée trois fois. Les autres espèces accompagnatrices sont $S$. cryophilum, $S$. auricoma et $S$. nitidifrons.

2) S. (S.) dorieri Doby et Rault 1960 .

La pauvreté de notre matériel (gîte 16: Cea, $650 \mathrm{~m}$ d'altitude) ne peut faire présumer de la fréquence de cette espèce à biologie encore assez mal connue.

Souvent récoltée en compagnie de $S$. monticola, elle est dans notre gîte associée à $S$. armoricanum et au groupe aureum.

\section{S. (S.) variegatum Meigen 1818 .}

Espèce banale de moyenne ou de basse altitude (Dorier 1962-1963), S. variegatum vit en général dans les mêmes biotopes que $S$. monticola à laquelle elle est souvent associée.

Déjà connue des principaux massifs montagneux de l'Espagne, nous l'avons récoltée dans quatre gîtes $(4,5,6$ et 7$)$, entre 250 et $1500 \mathrm{~m}$ d'altitude, dans la région des «Picos de Europa ». Très abondante dans les trois premiers prélèvements, elle s'y trouve associée à $S$. monticola (3 fois), $S$. nitidifrons et $S$. auricoma (1 fois).

\section{Conclusions}

Il est certain que la Péninsule ibérique n'a pas encore livrée toute sa richesse en ce qui concerne la faune simulidienne. En effet, dans cette région du Nord-Ouest de l'Espagne, à une époque assez peu favorable pour la récolte des simulies, en l'occurrence le début de l'automne, nous avons récolté 17 espèces dont 8 sont nouvelles pour ce pays.

$\mathrm{Si}$ nous nous appuyons sur les travaux de Rivosecchi (1971) concernant la biogéographie des simulies des massifs montagneux de l'Appennin Central, nous pouvons considérer que nous nous trouvons en présence:

- d'espèces orophiles, sténothermes d'eau froide: $S$. (E.) cryophilum, $S$. (S.) monticola, $S$. (S.) dorieri et $S$. (S.) variegatum;

- d'espèces orophiles mais eurythermes : $S$. bezzii et $S$. auricoma ;

- d'espèces d'eaux tempérées froides à répartition essentiellement septentrionale: $S$. (E.) armoricanum et $S$. (S.) naturale. La première n'était connue jusqu'à présent que de l'Ouest de la France (Massif armoricain) et de la Grande-Bretagne, la seconde de Grande-Bretagne uniquement;

- d'espèces d'eaux tempérées chaudes: $S$. $(W$.) mediterraneum et $S$. latinum, à vaste distribution méditerranéenne.

On ne peut préciser les affinités écologiques de $S$. (E.) latizonum, $S$. (E.) latigonium et des espèces du complexe «ornatum», encore mal connues sur le plan taxonomique. 


\section{Bibliographie}

B.illy-Choumara (H.), Bernard (M.-R.), Grenier (P.), Le Roy-Moret (M.-C.) et Mouchet (J.), 1970. - Note faunistique sur les Simulies (Diptera, Simuliidae) du Nord de la Tunisie. Cah. O.R.S.T.O.M., sér. Ent. méd. et Parasitol., 8, 377. 382.

Beaucournu-Saguez (F.), 1972. - Captures de Simulies dans la moitié sud du Portugal. Premières captures en Europe de Simulium sergenti Edwards 1923 et de Simulium ruficorne Macquart 1838. An. Esc. nac. Saude publ. e de Med. trop., 6, 73-83.

Beaucournu-Saguez (F.), 1975. - Récoltes de Simulies (Diptera, Simuliidae) dans le Sud-Est de l'Espagne. Bull. Soc. Entom. France, 79 (à paraître).

BEIRNE (B. P.), 1952. - The origin and history of british fauna, London.

Bernard (M. R.), 1970. - Simulium (Eusimulium) latinum (Rubzov, 1962) et Simulium (Simulium) maximum (Knoz, 1961) (Diptera, Simuliidae) espèces nouvelles pour la faune de France. Cah. O.R.S.T.O.M., sér. méd. Ent. et Parasitol., 8, 319-322.

Crosskey (R. W.), 1967. - A preliminary revision of the black-flies (Diptera: Simuliidae) of the Middle East. Trans. R. ent. Soc. Lond., 119, 1-45.

Crosskey (R.W.), 1970. - Simuliidae (Diptera) from the channel islands: first records. Entomologist's Gazette, 21, 125-132.

Crosskey (R. W.) et Davies (L.), 1972. - The identities of Simulium lineatum (Meigen), S. latipes (Meigen) and S. vernum Macquart (Diptera: Simuliidae). Entomologist's Gazette, 23, 249-258.

Davies (L.), 1966. - The taxonomy of British black-flies (Diptera: Simuliidae). Trans. R. ent. Soc. Lond., 118, 413-511.

Doby (J.-M.) et David (F.), 1960. - Présence en France (Dordogne, Pyrénées-Orientales, Haute-Savoie, Bas-Rhin, Pas-de-Calais, Manche et Côtes-du-Nord) de la forme spinosum Doby et Deblock, 1957, de Simulium ornatum Meigen, 1818 (Diptères-Simuliidés). Ann. Parasit. hum. comp., 35, 387-390.

Doby (J.-M.) et Deblock (S.), 1957. - Présence de tubercules tégumentaires épineux chez la nymphe d'une forme autrichienne de Simulium ornatum Meigen 1818, S. ornatum, f., spinosum n. f. Ann. Parasit. hum. comp., 32, 328-330.

Doby (J.-M.) et SAguez (F.), 1963. - Répartition géographique comparée des espèces de Simulies des Groupes monticola et latipes dans le Sud de la Grande-Bretagne et en Bretagne française. Bull. Soc. Pharm. Ouest, 3, 69-78.

DORIER (A.), 1962-1963. - Documents pour servir à la connaissance des Simuliidae du Sud-Est de la France. Trav. Lab. Hydrobiologie et de Pisciculture de l'Université de Grenoble, 54 et 55, 7-79.

Edwards (F.W.), 1933. - On three european species of Simulium. Ann. Naturhist. Museum Wien, 46, 255-256.

Giunicellı (J.), 1961. - Récoltes de Simuliidae (Diptera) en Corse. Etude faunistique et écologique. $86^{\circ}$ Congrès Soc. sav., 715-723. 
Giudicelli (J.), 1962. - Simulium galloprovinciale n. sp. (Diptera, Simuliidae), une simulie nouvelle du groupe auricoma. Comparaisons avec les espèces du genre Obuchovia Rubzov, 1951. Bull. Soc. Path. exot., 55, 882-892.

Grenier (P.) et Bertr 4ND (H.), 1949. - Simuliidae de la région orientale des Pyrénées. Complément à la description des différents stades de $S$. auricoma $\mathrm{Mg}$. Bull. Soc. Zool. France, 74, 294.

Grenier (P.) et Bertrand (H.), 1954. - Simuliidae (Diptera, Nematocera) d'Espagne. Ann. Parasit., hum. comp., 29, 447-459.

JARRY (D.), $1973 a$. - Contribution à l'étude des Simulies du Languedoc-Roussillon. I. Eusimulium latinum Rubzov et son écologie. Ann. Parasit. hum. comp., 48, 603-611.

JARRY (D.), $1973 \mathrm{~b}$. - Contribution à l'étude des Simulies du Languedoc-Roussillon. II. De quelques espèces des Pyrénées-Orientales. Ann. Parasit. hum. comp., 48, 703-712.

KNoz (J.), 1965. - To identification of Czechoslovakian black-flies (Diptera, Simuliidae). Folia, option Biologia, pp. $52+$ figures.

Moreau (R.E.), 1954. - The main vicissitudes of the European avifauna since Pliocene. Ibis, 96, 411-431.

RivosecchI (L.), 1962. - Contributo alla conoscenza dei simulidi italiani. III. Su qualche specie dei gruppi E. latipes (Meig.) ad E. aureum (Fries) raccolta nell'italia centrale e meridionale. Rivista di Parassitol., 23, 135-150.

RIvosecchi (L.), 1963. - Contributo alla conoscenza dei simulidi italiani. VIII. Due sottospecie dei gruppi monticola e latizonum. Riv. Parass., 24, 199-211.

Rivosecchi (L.), 1967. - I simulidi degli Appennini. Parassitologia, 9, 129-304.

RIVosecchi (L.), 1971. - Note biogeografiche sui simulidi (Diptera, Nematocera) dei massicci montuosi dell'appennino centrale. (XX. Contributo alla conoscenza dei simulidi italiani). Lavori della Soc. Ital. Biogeogr., II, 271-299.

Rivosecchi (L.) et Lipparoni (L.), 1964. - Contributo alla conoscenza dei Simulidi Italiani. $\mathrm{X}$. Comparazioni tra Odagmia pontica (Riv.), Odagmia ornata (Meig.) e Odagmia ornata nitidifrons (Edw.). Riv. Parassitol., 25, 249-268.

Rivosecchi (L.) et Solinas (A.), 1962. - Note e osservazioni. Nota preliminare sui Simulidi della Sardegna. Riv. Parassitol., 23, 309-312.

Rubzov (I. A.), 1956. - Faune de l'U.R.S.S., $\mathrm{n}^{\circ}$ 64, Diptera 6 (6) Simuliidae (en russe). Ac. Nauk. S.S.S.R., Moscou, 860 p.

Rubzov (I. A.), 1959-1964. - Simuliidae (Melusinidae) in Lindner E. Die Fleigen der Palaearktischen Region, B, III, 1-689.

Rubzov (I. A.), 1967. - Simuliidae d'Italia. Memoria II. Mem. Soc. Ent. Ital., 46, 127-180.

Serban (E.), 1951. - New and little-known species of black-flies of the group Eusimulium aureum Fries (Diptera, Simuliidae) from Rumania. Revue d'Entomologie de I'U.R.S.S., 40, 677-685.

Serra-Tosio (B.), 1962-1963. - Larves et nymphes de Simuliidae (Diptera) du bassin de l'Ardèche. Trav. Lab. Hydr. Pisc. Grenoble, 54 et 55, 123-142.

Zivnovic (V.), 1966. - Les Simulies (Diptera, Simuliidae) du groupe aureum en Yougoslavie. Acta vet. Beograd., 16, 257-264. 